\title{
Análisis de biomasa del vuelo de un rodal adulto de Pinus radiata
}

\author{
Analysis of aboveground biomass in a mature stand of Pinus radiata
}

\author{
CLAUDIO MUÑOZ RIVEROS, JORGE CANCINO C., MIGUEL ESPINOSA B. \\ Facultad de Ciencias Forestales, Universidad de Concepción, Casilla 160-C, Concepción, Chile. \\ E-mail: cmunozr@udec.cl
}

\begin{abstract}
SUMMARY
A study of biomass in Pinus radiata D. Don with 42 years old was made, the trees were obtained of a stand placed between the cities of Santa Juana and Nacimiento, VIII Region, Chile. Three classes of top were differentiated in the stand: dominant, codominant and intermediate. The value of the mass for each component (wood, bark, branches, branchlets and needle) was obtained from each class of top; the wood resulted as the most important component in the total biomass of the tree $(88.9 \%)$, then the bark $(8.2 \%)$ and branches $(1.5 \%)$. Obtained the values of biomass, the function of biomass for each component was obtained, related this values with the variables of the tree; the variables that showed greater correlation with the values of biomass by component and minors estimation errors are dbh (breast height diameter) and dbc (base of live crown diameter).
\end{abstract}

Key words: Arboreal biomass, Pinus radiata, predictors models of biomass, analysis of biomass, distribution of arboreal biomass by components.

\section{RESUMEN}

Se realizó un estudio de biomasa en árboles de Pinus radiata D. Don de 42 años, obtenidos de un rodal ubicado entre las ciudades de Santa Juana y Nacimiento, VIII Región, Chile.

El rodal se diferenció en tres clases de copa: dominante, codominante e intermedio. En cada clase de copa se obtuvieron valores de biomasa para cada componente (madera, corteza, ramas, ramillas y acículas); la madera resultó ser el componente que más aportó a la biomasa total sobre el suelo $(88,9 \%)$, seguido de la corteza $(8,2 \%)$ y ramas $(1,5 \%)$.

Con los valores de biomasa obtenidos, se ajustaron funciones de biomasa por componente, relacionando estos valores con variables del árbol; el dap (diámetro a la altura del pecho) y el dbc (diámetro en la base de la copa viva) fueron las variables que mostraron mayor correlación con los valores de biomasa por componente y menores errores de estimación.

Palabras clave: Biomasa arbórea, Pinus radiata, modelos predictores de biomasa, análisis de biomasa, distribución de biomasa arbórea por componentes.

\section{INTRODUCCION}

La biomasa forestal se define como la masa de todos los componentes arbóreos, excepto tocón y raíces, en una superficie dada. Este parámetro es el que mejor expresa la productividad de un sistema biológico. Desde la perspectiva forestal, la masa seca del material leñoso está más relacionada con el rendimiento de material astillable, pulpable y combustible que los parámetros habitualmente medidos en inventarios de madera en pie (1). Referido a un árbol, la biomasa se define como la masa del ejemplar por sobre el nivel del suelo o corta; puede ser total o separado entre los componentes principales como son el fuste, ramas y follaje. A su vez, la biomasa 
del fuste puede referirse a un diámetro límite de utilización (2).

Desde el punto de vista energético, la biomasa puede volver a cubrir una parte importante de nuestras necesidades energéticas, al considerar las ventajas de su uso: no efecto invernadero, sustitución de actividades agrícolas ahora excedentarias, aprovechamiento de residuos (3), diversificación del suministro de energía (producción de etanol desde biomasa lignocelulósica) (4), etc. Además, los bosques de coníferas en particular constituyen la mayor reserva de carbono y su contribución a la mitigación en los cambios climáticos es reconocida por su capacidad de capturar el dióxido de carbono (5) desde la atmósfera a través de la fotosíntesis y por su gran capacidad de almacenamiento en componentes bióticos y abióticos (6).

$\mathrm{Al}$ establecer un bosque con fines productivos, particularmente en lo que a producción de madera se refiere, el principal objetivo es maximizar su productividad, lo que implica un óptimo aprovechamiento de la potencialidad del sitio, cuidando la sustentabilidad del mismo. Para manejar eficientemente la productividad, es necesario cuantificar la productividad potencial, identificar qué rodales tienen una producción bajo el potencial, determinar qué limitaciones del suelo y/o rodal son responsables de los niveles de producción bajo el óptimo y desarrollar tratamientos silviculturales que mejoran efectivamente las limitaciones que han sido identificadas (7). Otro importante punto es cómo las plantaciones pueden crecer por un número indefinido de rotaciones sin afectar la capacidad del sitio para producción de biomasa (8).

La medición directa de la masa de un árbol, ya sea total o por componente (madera, corteza, ramas, ramillas y acículas), es un proceso difícil y costoso, por lo que es más conveniente realizar una estimación de la masa seca de cada componente. Esa estimación se realiza a partir de submuestras consistentes en rodelas, ramas, ramillas y otros, obtenidos de árboles seleccionados según algún criterio. La masa seca de cada componente del árbol se estima usando fracciones de masa seca/masa verde de las submuestras obtenidas (9), o mediante funciones ajustadas con esa información $(10,11)$.

Uno de los factores más importantes que contribuye al desarrollo de un rodal es la cantidad de biomasa foliar. Aunque su contribución al total de la biomasa arbórea es de sólo 4 a 6 por ciento, la biomasa foliar es la responsable de los procesos de fotosíntesis-transpiración y de la captación total de carbono en el árbol (12). La biomasa foliar es además muy sensitiva a patrones climáticos y tratamientos silviculturales, por lo que la cuantificación de la biomasa foliar puede ser importante para explicar la productividad de rodales.

El objetivo general de este estudio es la determinación de la biomasa por árbol y por hectárea de un rodal adulto de Pinus radiata D. Don., la especie arbórea con mayor superficie plantada en Nueva Zelanda (13) y Chile.

Entre los objetivos específicos está determinar la participación de cada componente en la biomasa seca total sobre el suelo y comparar la participación de cada componente entre clases de copa. A su vez, se busca ajustar funciones predictoras de biomasa por componente y total utilizando variables de estado de fácil obtención en terreno; determinar la distribución de la masa seca de madera y corteza a lo largo del fuste.

\section{MATERIAL Y METODOS}

Descripción del área de estudio. La información utilizada en el estudio se recolectó en un rodal del Fundo Monterrey, ubicado entre las ciudades de Santa Juana y Nacimiento, a $83 \mathrm{~km}$ al sudeste de la ciudad de Concepción, VIII Región, Chile.

La precipitación media anual en el área de estudio es de $1.093 \mathrm{~mm}$, con una distribución de $295 \mathrm{~mm}$ en otoño $(27 \%), 553 \mathrm{~mm}$ en invierno $(51 \%), 171 \mathrm{~mm}$ en primavera (16\%) y sólo 74 $\mathrm{mm}$ en verano $(6 \%)$. La temperatura media anual es de $15,4^{\circ} \mathrm{C}$, alcanzando la media máxima en enero a $28,6^{\circ} \mathrm{C}$, con una media en enero de $19,0^{\circ} \mathrm{C}$ $\mathrm{y}$ en julio de $4,4^{\circ} \mathrm{C}$. El lugar presenta períodos de sequía estival que comprenden cinco meses por año (14).

El rodal en estudio creció en un suelo de textura arenosa (serie Arenales), con una topografía que presenta pendientes que van desde $7 \%$ a $43 \%$, aproximadamente.

Descripción del rodal en estudio. El rodal tenía 42 años al momento de su cosecha, oportunidad en la que se realizó el muestreo para efectos de este estudio. El rodal abarcaba una superficie de 8,3 hectáreas, con una densidad de 315 árboles por hectárea y $59,2 \mathrm{~m}^{2} / \mathrm{ha}$ de área basal. 
El rodal fue sometido a manejo silvícola (poda y raleo), efectuándose dos podas, la primera a una altura de 2,5 m cuando los árboles tenían aproximadamente ocho años de edad. La segunda poda fue realizada aproximadamente a los 13 años, fluctuando la altura de poda entre 9,3 m y $12 \mathrm{~m}$. Si bien no se tiene información respecto a la fecha de ejecución de los raleos, se cree que se realizaron dos raleos comerciales, a juzgar por la densidad final del rodal, los que no habrían sido ejecutados en forma oportuna por la estructura del rodal.

La calidad de los árboles era regular, pues aproximadamente sólo un tercio de ellos poseían fustes rectos y bien formados, predominando los árboles inclinados, de copas irregulares y con defectos a lo largo del fuste, tales como pudriciones y cancros.

Tamaño de la muestra. El rango de valores de dap (diámetro a la altura del pecho) del rodal fue dividido en tres clases de igual amplitud y se asignó a éstas equivalencia a las clases de copa intermedio, codominante y dominante (15). Cada clase de dap se subdividió en cuatro intervalos y en cada uno de ellos se seleccionó un árbol de dap cercano a la marca de clase del intervalo (cuadro 1). Así, la muestra quedó constituida por 12 árboles, todos los cuales fueron utilizados para la estimación de los componentes de copa; uno de ellos se descartó para la estimación de los componentes fustales.

\section{CUADRO 1}

Dap de los árboles muestra y frecuencia por clase de copa.

Dbh of the trees sample and frequency for class of crown.

\begin{tabular}{|lccc|}
\hline Clase de copa & dap (cm) & \multicolumn{2}{c|}{$\begin{array}{c}\text { Densidad } \\
\text { (árboles/ha) }\end{array}$} \\
& & $(\%)$ \\
\hline Dominante & $57,0-60,4-63,2-65,6$ & 82,5 & 26,2 \\
Codominante & $43,5-46,5-50,3-54,3$ & 115,0 & 36,5 \\
Intermedio & $29,5-33,1-37,7-41,2$ & 117,5 & 37,3 \\
Total & & 315,0 & 100,0 \\
\hline
\end{tabular}

Obtención de datos de terreno. Los árboles seleccionados fueron marcados y enumerados, midiendo en cada uno de ellos el radio de copa hacia los cuatro puntos cardinales, el dap y diámetro a los
$30 \mathrm{~cm}$ de altura. Luego se procedió al volteo y medición de las siguientes variables: espesor de corteza a la altura del dap, altura hasta los diámetros límite de utilización comercial (dlu) 10 y 20 $\mathrm{cm}$, altura total (incluyendo longitud del tocón), longitud de copa viva, diámetro en la base de la copa viva y altura del tocón (figura 1).

A continuación se procedió al desrame y medición de la masa de la copa viva del árbol, mediante un dinamómetro (precisión $250 \mathrm{~g}$ ). La copa viva, delimitada entre los verticilos de la base de la copa viva y el último verticilo visible, fue dividida en cuatro secciones de similar longitud (16). De cada sección se seleccionaron al azar tres ramas (submuestra de ramas) (16), las que fueron etiquetadas y guardadas en bolsas plásticas para su posterior análisis en laboratorio (figura 1).

Posteriormente se obtuvieron rodelas en el tocón y a alturas variables según la calidad y largo comercial de la troza. Producto de la caída, la parte superior de los fustes se quebró, por lo que la última rodela se obtuvo a la mayor altura posible antes del quiebre del fuste. El espesor de las rodelas fluctuó entre 2 y $4 \mathrm{~cm}$ y éstas fueron etiquetadas, guardadas en bolsas plásticas y almacenadas a $2^{\circ} \mathrm{C}$ en cámaras de frío para su posterior análisis $(16,17)$ (figura 1).

Obtención de datos en laboratorio. En laboratorio se midió la masa verde de las muestras de ramas seleccionadas de cada sección de la copa viva del árbol; se midió la masa total de cada rama y la masa de madera, ramillas y acículas por separado (18), medición realizada en una balanza electrónica (precisión 0,01 g). De todas las ramillas se seleccionó una submuestra de seis ramillas, en las que se midió la masa de ramillas y de acículas. Posteriormente se secaron en horno a $100^{\circ} \mathrm{C}$ durante 24 horas, o hasta que alcanzara masa constante, y luego se pesaron en seco (16) (figura 1).

A las rodelas obtenidas de cada árbol se les determinó la masa verde y la gravedad específica a partir de la relación entre la masa y el volumen (obtenido por desplazamiento de agua en un recipiente graduado) (16), tanto en verde como en seco. También se determinó el diámetro dentro y fuera de la corteza. Luego las rodelas fueron secadas en horno a $100^{\circ} \mathrm{C}$ durante 24 horas o hasta que alcanzaron masa constante (17). Se 


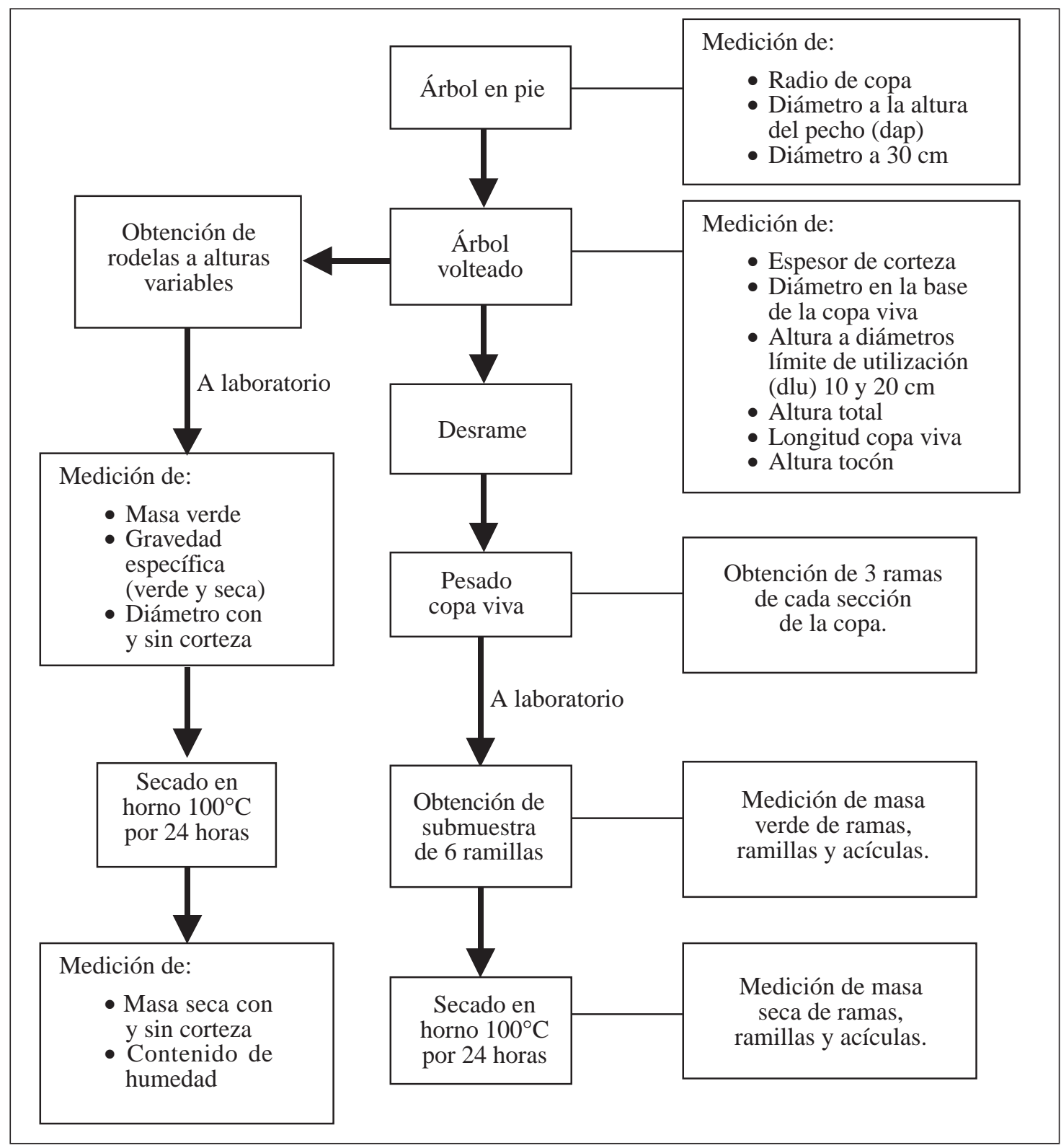

Figura 1. Flujo de actividades de obtención de datos en terreno y en laboratorio.

Flow of activities of obtaining data in land and laboratory.

determinó así la masa seca de la rodela con corteza y luego descortezada, para obtener la masa seca de madera y el contenido de humedad de cada rodela (figura 1).

Estimación de la masa seca fustal. Primero se determinó la densidad verde de la madera, relacionando la masa verde de cada rodela con su volumen respectivo, obtenido por desplazamiento de agua en un recipiente graduado.

$$
d_{v}=M_{v} / V_{v}
$$

donde:

$d_{v}$ : Densidad verde de la madera

$M_{v}$ : Masa verde de la rodela

$V_{v}$ : Volumen verde de la rodela

A continuación se obtuvo la masa verde de cada sección fustal a partir del volumen y la densidad verde de cada sección. El volumen total de 
cada sección fue calculado a partir de la longitud de cada sección fustal y de los diámetros de las rodelas de los extremos de cada sección, asumiendo la forma de un paraboloide (Fórmula de Smalian) (19).

$M_{i}=V_{i} *\left(\left(d_{i b}+d_{i s}\right) / 2\right)$, para las

secciones centrales

donde:

$M_{i}$ : Masa verde de la i-ésima sección fustal

$V_{i}$ : Volumen de la i-ésima sección fustal

$d_{i b}$ : Densidad verde de la rodela en la base de la i-ésima sección fustal

$d_{i s}$ : Densidad verde de la rodela en el extremo superior de la i-ésima sección fustal

Tanto en el sector del tocón como en la sección fustal más próxima al ápice, se asumió $\mathrm{d}_{i b}=\mathrm{d}_{i s}$.

Luego, se obtuvo la fracción de masa seca para madera y corteza por rodela a partir de las variables respectivas.

$$
\begin{gathered}
F m s_{i}=M s m_{i} / M v t_{i} \\
F c s_{i}=M s c_{i} / M v t_{i}
\end{gathered}
$$

donde:

$F m s_{i}$ : Fracción de madera seca de la rodela i $F_{C s_{i}}$ : Fracción de corteza seca de la rodela i $M_{\mathrm{Sm}}$ : Masa seca de madera de la rodela i $M s c_{i}$ : Masa seca de corteza de la rodela i $M v t_{i}$ : Masa verde total (madera + corteza) de la rodela $\mathrm{i}$

Finalmente se estimó la masa seca fustal de madera y corteza por árbol, a partir de las fracciones promedio por sección fustal.

$$
\begin{gathered}
M S M=\sum_{i=1}^{n}\left[\left(F m s_{i b}+F m s_{i s}\right) / 2\right] \times M_{i} \\
M S C=\sum_{i=1}^{n}\left[\left(F c s_{i b}+F c s_{i s}\right) / 2\right] \times M_{i}
\end{gathered}
$$

donde:

MSM : Masa seca de madera fustal del árbol $M S C$ : Masa seca de corteza fustal del árbol

$F m s_{i b}$ : Fracción de madera seca en la rodela de la base de la i-ésima sección fustal
$F m s_{i s}$ : Fracción de madera seca en la rodela del extremo superior de la i-ésima sección fustal

$F c s_{i b}$ : Fracción de corteza seca en la rodela de la base de la i-ésima sección fustal

$F_{c s}$ is : Fracción de corteza seca en la rodela del extremo superior de la i-ésima sección fustal

$\mathrm{M}_{\mathrm{i}} \quad$ : Masa verde total (madera + corteza) de la i-ésima sección fustal

$\mathrm{n} \quad$ : Número de secciones en el fuste

Estimación de la masa seca de la copa. La masa seca de la copa se obtuvo a partir de las fracciones promedio de masa seca por componente y sección (16). El promedio de cada fracción se obtuvo de las tres ramas seleccionadas de cada sección (cuadro 2).

Análisis de los datos. Con la información recopilada se determinó la participación porcentual de cada componente respecto a la biomasa total del árbol. Se determinó la distribución de masa seca de madera y corteza del fuste y su variación con la altura relativa del árbol. Se realizaron comparaciones de los valores de biomasa entre clases de copa, asumiendo un diseño completamente aleatorio (20).

Posteriormente se procedió al ajuste de relaciones entre la biomasa por componente (madera, corteza, ramas, ramillas y acículas) y variables obtenidas en terreno como diámetro a la altura del pecho, altura total (5), longitud de copa viva, diámetro en la base de la copa viva y diámetro a la altura del tocón. Para seleccionar la mejor función se consideraron los valores del coeficiente de determinación ajustado $\left(\mathrm{R}^{2}\right)$, el error estándar de estimación (EEE) y la prueba F. Cada variable debía aportar al menos un cinco por ciento de la variación.

\section{RESULTADOS Y DISCUSION}

Dimensiones medias de los árboles. Los árboles dominantes son significativamente mayores en dap, espesor de corteza, área basal, altura y volumen que los árboles codominantes e intermedios. Sólo en el espesor de corteza y altura total no presentan diferencia significativa con los árboles codominantes y en longitud de copa viva con los árboles codominantes e intermedios (cuadro 3). 


\section{CUADRO 2}

Fórmulas utilizadas para la estimación de la masa seca de los componentes de la copa.

Formulas used for the estimation of the dry mass of the components of the crown.

\begin{tabular}{|c|c|c|c|}
\hline Masa seca & Fórmula & & Definición \\
\hline \multirow{3}{*}{$\begin{array}{c}\text { Madera de la copa } \\
\text { del árbol (MST) }\end{array}$} & $M S T=\sum_{i=1}^{k}$ & [7] & $\begin{array}{l}F t s_{i}: \text { Fracción promedio de madera seca de } \\
\text { rama de la sección i de la copa. } k \text { : Número } \\
\text { de secciones de la copa. } M_{i}: \text { Masa verde } \\
\text { total (tronco+ramillas+acículas) de la } \\
\text { sección i de la copa. }\end{array}$ \\
\hline & $F t s_{i}=\sum_{j=1}^{n r} F t s_{j} / n r$ & [8] & $\begin{array}{l}\text { Fts } s_{j} \text { : Fracción de madera seca de la rama j. } \\
n r: \text { Número de ramas muestreadas en la } \\
\text { sección i de la copa. }\end{array}$ \\
\hline & $F t s_{j}=M s t_{j} / M v t_{j}$ & [9] & $\begin{array}{l}M s t_{j}: \text { Masa seca de madera de la rama } \mathrm{j} \\
M v t_{j}: \text { Masa verde total } \\
\text { (madera+ramillas+acículas) de la rama j. }\end{array}$ \\
\hline \multirow{3}{*}{$\begin{array}{l}\text { Ramillas de la copa } \\
\text { del árbol (MSR) }\end{array}$} & $M S R=\sum_{j=1}^{k} \operatorname{Frs}_{i} \times M_{i}$ & {$[10]$} & $\begin{array}{l}F r s_{i} \text { : Fracción promedio de ramillas secas } \\
\text { de rama de la sección i de la copa. }\end{array}$ \\
\hline & $F r s_{i}=\sum_{j=1}^{n r} F r s_{j} / n r$ & [11] & Frs: Fracción de ramillas secas de la rama j. \\
\hline & $F r s_{j}=M s r_{j} / M v t_{j}$ & {$[12]$} & $M s r_{j}:$ Masa seca de ramillas de la rama $\mathrm{j}$ \\
\hline \multirow{3}{*}{$\begin{array}{l}\text { Acículas de la copa } \\
\text { del árbol (MSA) }\end{array}$} & $M S A=\sum_{i=1}^{k} \operatorname{Fas}_{i} \times M_{i}$ & [13] & $\begin{array}{l}\text { Fas: Fracción promedio de acículas secas } \\
\text { de rama de la sección i de la copa. }\end{array}$ \\
\hline & Fas $_{i}=\sum_{j=1}^{n r}$ Fas $_{j} / n r$ & {$[14]$} & Fas: Fracción de acículas secas de la rama j. \\
\hline & $\mathrm{Fas}_{j}=M s a_{j} / M v t_{j}$ & {$[15]$} & $M s a_{j}:$ Masa seca de acículas de la rama j. \\
\hline
\end{tabular}

Los árboles codominantes ocupan el segundo lugar entre clases de copa en la mayoría de las variables medidas, siendo la excepción la clase de forma de Girard y la razón de copa viva. En casi todas las variables, los valores promedio de esta clase de copa estuvieron más cerca de los valores promedio de la clase de copa dominante que de los intermedios, a excepción de la longitud de copa viva (cuadro 3 ).

Consecuente con su posición subordinada en el dosel de copas, los árboles intermedios presentan la mayor razón altura/dap (coeficiente de esbeltez) y la menor razón de copa viva. Además poseían copas significativamente más estrechas que los árboles dominantes (cuadro 3).

Análisis de volumen y de la biomasa total y por componente. El volumen promedio por hectárea determinado en árboles dominantes fue $458 \mathrm{~m}^{3}$, en árboles codominantes $391 \mathrm{~m}^{3}$ y $188 \mathrm{~m}^{3}$ en árboles intermedios. El volumen total por hectárea fue $1.037 \mathrm{~m}^{3}$, de los cuales $1.032 \mathrm{~m}^{3}$ corresponden a volumen hasta dlu $10 \mathrm{~cm}$ y $979 \mathrm{~m}^{3}$ a volumen hasta dlu $20 \mathrm{~cm}$. Gerding y Schlatter (21) demostraron que la productividad varía ampliamente dentro de cada zona climática y tipo de 


\section{CUADRO 3}

Valores promedio de variables características de los árboles muestra.

Average values of characteristics variables of the trees of the sample.

\begin{tabular}{|c|c|c|c|c|c|c|}
\hline \multirow[b]{3}{*}{ Variable característica } & \multicolumn{6}{|c|}{ Clase de copa } \\
\hline & \multicolumn{2}{|c|}{ Intermedio $(n=4)$} & \multicolumn{2}{|c|}{ Codominante $(n=4)$} & \multicolumn{2}{|c|}{ Dominante $(n=4)$} \\
\hline & Promedio & EEM & Promedio & $\overline{\mathrm{EEM}}$ & Promedio & EEM \\
\hline \multicolumn{7}{|l|}{ Diámetro $(\mathrm{cm})$ : } \\
\hline Tocón & $43,5 \mathrm{a}$ & 3,4 & $55,3 \mathrm{~b}$ & 2,6 & $77,0 \mathrm{c}$ & 0,8 \\
\hline dap (c.c.) & $35,4 \mathrm{a}$ & 2,6 & $48,6 \mathrm{~b}$ & 2,3 & $61,6 \mathrm{c}$ & 1,8 \\
\hline dap (s.c.) & $30,4 a$ & 2,5 & $42,3 b$ & 2,3 & $54,0 \mathrm{c}$ & 1,8 \\
\hline Espesor corteza $(\mathrm{cm})$ & $2,5 \mathrm{a}$ & 0,1 & $3,1 \mathrm{ab}$ & 0,4 & $3,8 b$ & 0,1 \\
\hline Area basal $\left(\mathrm{m}^{2}\right)$ & $0,1 \mathrm{a}$ & 0,0 & $0,2 b$ & 0,0 & $0,3 \mathrm{c}$ & 0,0 \\
\hline \multicolumn{7}{|l|}{ Altura (m): } \\
\hline Total árbol & $37,2 \mathrm{a}$ & 2,3 & $45,0 \mathrm{~b}$ & 0,6 & $49,8 b$ & 1,4 \\
\hline Longitud copa & $13,0 \mathrm{a}$ & 3,4 & $15,0 \mathrm{a}$ & 1,6 & $20,6 \mathrm{a}$ & 1,9 \\
\hline \multicolumn{7}{|l|}{ Volumen $\left(\mathrm{m}^{3}\right)$ : } \\
\hline Total fuste & $1,6 \mathrm{a}$ & 0,3 & $3,4 b$ & 0,3 & $5,6 \mathrm{c}$ & 0,3 \\
\hline dlu $10 \mathrm{~cm}$ & $1,6 \mathrm{a}$ & 0,3 & $3,4 \mathrm{~b}$ & 0,3 & $5,6 \mathrm{c}$ & 0,3 \\
\hline dlu $20 \mathrm{~cm}$ & $1,3 \mathrm{a}$ & 0,3 & $3,2 b$ & 0,3 & $5,4 \mathrm{c}$ & 0,4 \\
\hline Clase de Forma Girard & $78,5 \mathrm{a}$ & 0,7 & $78,0 \mathrm{a}$ & 0,3 & $80,0 \mathrm{a}$ & 1,2 \\
\hline \multicolumn{7}{|l|}{ Razones: } \\
\hline Altura/dap & $1,1 \mathrm{a}$ & 0,0 & $0,9 \mathrm{ab}$ & 0,0 & $0,8 b$ & 0,0 \\
\hline Long. Copa/altura & $0,3 \mathrm{a}$ & 0,1 & $0,3 \mathrm{a}$ & 0,0 & $0,4 \mathrm{a}$ & 0,0 \\
\hline
\end{tabular}

EEM: Error estándar de la media.

$\mathrm{n}$ : Tamaño de la muestra.

Signif.: Letras diferentes indican que existe diferencia significativa entre clases de copa ( $<<0,05$, Test Tukey).

suelo. Sus resultados sugieren que debiera haber una alta probabilidad de incrementar la productividad, desde el punto de vista biológico y económico, en sitios que presentan una productividad inferior al potencial para la clase de sitio en que se encuentran.

La biomasa total promedio por árbol dominante (cuadro 4) alcanzó a $2.090 \mathrm{~kg}$, lo que equivale a 1,5 veces la biomasa de los árboles codominantes $(1.388 \mathrm{~kg})$ que a su vez poseen 2,2 veces la biomasa de los árboles intermedios (627 $\mathrm{kg}$ ). Los árboles dominantes tienen en promedio 3,3 veces más biomasa que los árboles intermedios. Rodríguez et al. (17) comparando rodales de Pinus radiata sometidos a regímenes silvopastoral y tradicional, encontraron que la biomasa total por árbol bajo el régimen silvopastoral fue 2,1 a 2,5 veces mayor que en el régimen convencional. Sin embargo, la distribución de la biomasa sobre el suelo no se vio afectada por el régimen silvicultural ni por los esquemas de manejo silvopastoral.

La biomasa total por hectárea en los árboles dominantes alcanzó a 172 toneladas; en tanto que en árboles codominantes alcanzó a 159 toneladas y 74 toneladas en árboles intermedios. En suma, las tres clases de copa totalizan 405 toneladas por hectárea (cuadro 5).

Para las tres clases de copa, la madera aporta en promedio $89 \%$ de la biomasa total sobre el suelo, la corteza $8,2 \%$, ramas $1,5 \%$, acículas $0,8 \%$ y ramillas $0,7 \%$. En las tres clases de copa, la madera concentra alrededor del $90 \%$ de la biomasa total sobre el suelo. Las diferencias porcentuales entre clases de copa son mínimas y no significativas (cuadro 4).

Lo anterior coincide con lo encontrado por Helmisaari et al. (18), quienes concluyeron que la 


\section{CUADRO 4}

Biomasa promedio (kg/árbol) y participación de cada componente (\%) por clase de copa. Average of biomass (kg/tree) and participation of each component (\%) for class of crown.

\begin{tabular}{|c|c|c|c|c|c|c|c|c|c|}
\hline \multirow[b]{3}{*}{ Componente } & \multicolumn{9}{|c|}{ Clase de Copa } \\
\hline & \multicolumn{3}{|c|}{ Intermedio } & \multicolumn{3}{|c|}{ Codominante } & \multicolumn{3}{|c|}{ Dominante } \\
\hline & $\begin{array}{c}\text { Masa } \\
\text { (kg/árbol) }\end{array}$ & EEM & $(\%)$ & $\begin{array}{c}\text { Masa } \\
\text { (kg/árbol) }\end{array}$ & EEM & $(\%)$ & $\begin{array}{c}\text { Masa } \\
\text { (kg/árbol) }\end{array}$ & EEM & $(\%)$ \\
\hline Madera fuste & $549,9 a$ & 62,3 & 87,7 & $1224,5 b$ & 83,5 & 88,2 & $1880,4 \mathrm{c}$ & 161,5 & 90,0 \\
\hline Corteza fuste & $62,4 \mathrm{a}$ & 3,4 & 10,0 & $129,6 b$ & 15,6 & 9,3 & $132,0 \mathrm{~b}$ & 10,8 & 6,3 \\
\hline Total fuste & $612,3 a$ & 64,0 & 97,7 & $1354,1 \mathrm{~b}$ & 93,9 & 97,5 & $2012,5 b$ & 171,3 & 96,3 \\
\hline Acículas & $5,2 \mathrm{a}$ & 1,8 & 0,8 & $9,5 \mathrm{ab}$ & 1,8 & 0,7 & $18,1 \mathrm{~b}$ & 2,5 & 0,9 \\
\hline Ramillas & $3,9 \mathrm{a}$ & 1,5 & 0,6 & $8,3 \mathrm{a}$ & 1,3 & 0,6 & $15,4 \mathrm{~b}$ & 1,5 & 0,7 \\
\hline Ramas & $5,9 \mathrm{a}$ & 1,9 & 0,9 & $14,1 \mathrm{~b}$ & 2,6 & 1,0 & $44,4 \mathrm{c}$ & 6,7 & 2,1 \\
\hline Total copa $*$ & $83,6 \mathrm{a}$ & 45,5 & 13,3 & $118,6 \mathrm{a}$ & 31,8 & 8,5 & $310,3 a$ & 81,0 & 14,8 \\
\hline Total árbol ** & $627,3 \mathrm{a}$ & 68,4 & 100 & $1388,0 \mathrm{~b}$ & 99,4 & 100 & $2090,4 \mathrm{c}$ & 178,4 & 100 \\
\hline
\end{tabular}

EEM: Error estándar de la media.

Signif.: Letras diferentes indican que existe diferencia significativa entre clases de copa $(\mathrm{P}<0,05$, Test Tukey).

$\%$ : Porcentaje de participación del componente respecto al total del árbol sobre el suelo.

*: La masa seca total de la copa incluye la sección del fuste de la copa.

**: Masa seca total del árbol sobre el suelo.

\section{CUADRO 5}

Biomasa de cada componente por hectárea (t/ha) y participación porcentual de cada componente por clase de copa respecto al total por hectárea.

Biomass of each component per hectare (t/ha) and percentual participation of each component for class of crown regarding the total per hectare.

\begin{tabular}{|c|c|c|c|c|c|c|c|c|c|c|c|}
\hline \multirow[b]{3}{*}{ Componente } & \multicolumn{9}{|c|}{ Clase de Copa } & \multirow{2}{*}{\multicolumn{2}{|c|}{ Total Hectárea }} \\
\hline & \multicolumn{3}{|c|}{ Intermedio } & \multicolumn{3}{|c|}{ Codominante } & \multicolumn{3}{|c|}{ Dominante } & & \\
\hline & $\begin{array}{c}\text { Masa } \\
(\mathrm{t} / \mathrm{ha})\end{array}$ & EEM & $(\% 1)$ & $\begin{array}{l}\text { Masa } \\
(\mathrm{t} / \mathrm{ha})\end{array}$ & EEM & $(\% 1)$ & $\begin{array}{c}\text { Masa } \\
(\mathrm{t} / \mathrm{ha})\end{array}$ & EEM & $(\% 1)$ & $\begin{array}{l}\text { Masa } \\
(\mathrm{t} / \mathrm{ha})\end{array}$ & $(\% 2)$ \\
\hline Madera fuste & $64,6 \mathrm{a}$ & 7,3 & 17,9 & $140,8 b$ & 9,6 & 39,0 & $155,1 \mathrm{~b}$ & 13,3 & 43,1 & 360,5 & 88,9 \\
\hline Corteza fuste & $7,3 \mathrm{a}$ & 0,4 & 22,1 & $14,9 \mathrm{~b}$ & 1,8 & 45,0 & $10,9 b$ & 0,9 & 32,9 & 33,1 & 8,2 \\
\hline Total fuste & $71,9 \mathrm{a}$ & 7,5 & 18,3 & $155,7 \mathrm{~b}$ & 10,8 & 39,6 & $166,0 \mathrm{~b}$ & 14,1 & 42,1 & 393,6 & 97,1 \\
\hline Acículas & $0,6 \mathrm{a}$ & 0,2 & 19,0 & $1,1 \mathrm{ab}$ & 0,2 & 34,2 & $1,5 b$ & 0,2 & 46,8 & 3,2 & 0,8 \\
\hline Ramillas & $0,5 \mathrm{a}$ & 0,2 & 17,2 & $1,0 \mathrm{ab}$ & 0,1 & 35,5 & $1,3 b$ & 0,1 & 47,3 & 2,7 & 0,7 \\
\hline Ramas & $0,7 \mathrm{a}$ & 0,2 & 11,5 & $1,6 \mathrm{~b}$ & 0,2 & 27,2 & $3,7 \mathrm{c}$ & 0,6 & 61,3 & 6,0 & 1,5 \\
\hline Total copa * & $9,8 \mathrm{a}$ & 5,3 & 20,0 & $13,6 \mathrm{a}$ & 3,6 & 27,8 & $25,6 \mathrm{a}$ & 6,7 & 52,2 & 49,1 & 12,1 \\
\hline Total árbol ** & $73,7 \mathrm{a}$ & 8,0 & 18,2 & $159,4 b$ & 11,4 & 39,3 & $172,5 \mathrm{a}$ & 14,7 & 42,5 & 405,6 & \\
\hline
\end{tabular}

EEM: Error estándar de la media.

Signif.: Letras diferentes indican que existe diferencia significativa entre clases de copa ( $\mathrm{P}<0,05$, Test Tukey).

\%: Porcentaje de participación del componente por clase de copa respecto al total por hectárea.

\%2: Porcentaje de participación del componente respecto al total del árbol sobre el suelo.

*: La masa seca total de la copa incluye la sección del fuste de la copa.

**: Masa seca total del árbol sobre el suelo. 
madera fue el principal componente de la biomasa para un bosque adulto (35 años) y para un bosque maduro (100 años) de pino escocés (Pinus sylvestris L.) en el este de Finlandia. No obstante, las ramas fueron el principal componente de la biomasa en renovales de 15 años de la misma especie.

En tanto que los árboles dominantes son los que aportan un mayor porcentaje de madera por hectárea debido al mayor número de árboles de esta clase de copa. En suma, las tres clases de copa aportan 361 toneladas por hectárea de madera, lo que equivale al $89 \%$ de la biomasa total por hectárea (cuadro 5). Los árboles dominantes presentan menor porcentaje de corteza $(6,3 \%)$ que los árboles codominantes $(9,3 \%)$ e intermedios $(10,0 \%)$ (cuadro 4). Los árboles codominantes presentan mayor porcentaje de masa seca de corteza por hectárea $(45,0 \%)$ que los árboles dominantes $(32,9 \%)$ e intermedios $(22,1 \%)$ (cuadro 5).

Las ramas de los árboles dominantes representan el mayor porcentaje respecto de la biomasa total $(2,1 \%)$ en comparación a los árboles codominantes $(1,0 \%)$ e intermedios $(0,9 \%)$ (cuadro 4). Además, los árboles dominantes aportaron el mayor porcentaje de masa seca de ramas por hectárea, representando el $61,3 \%$ de la masa seca total, seguido de los árboles codominantes $(27,2 \%)$ y los árboles intermedios (11,5\%) (cuadro 5).
Las ramillas son el componente que menos aporta a la biomasa total sobre el suelo, con porcentajes que no superan el $1 \%$ para las tres clases de copa. La masa seca de ramillas por hectárea de los árboles dominantes alcanza a 1,3 toneladas (47,3\% del total por hectárea del rodal), cifra que es significativamente superior a la de árboles codominantes (1 tonelada, 35,5\%) e intermedios (0,5 toneladas, $17,2 \%$ ) (cuadro 5). Por su parte, las acículas también representan menos del $1 \%$ de la biomasa total sobre el suelo, en las tres clases de copa.

Los árboles dominantes representan el mayor porcentaje de masa seca de acículas por hectárea $(46,8 \%)$, por sobre los árboles codominantes $(34,2 \%)$ e intermedios (19\%) (cuadro 5). Estos resultados están directamente relacionados con la producción de biomasa del árbol, ya que a mayor área foliar, mayor absorción de radiación solar y, por tanto, mayor producción de biomasa (7).

Distribución de la masa seca del fuste según altura relativa en el árbol. El 80,2\% de la masa seca total de madera se concentra en la primera mitad del fuste (figura 2). Los árboles dominantes concentran un porcentaje de masa seca levemente superior a las otras clases de copa, pero en general el comportamiento es muy similar en las tres clases de copa (cuadro 6).

\section{CUADRO 6}

Distribución de biomasa por clase de copa y promedio según altura relativa.

Distribution of biomass for class of crown and average according to relative height.

\begin{tabular}{|c|c|c|c|c|c|c|c|c|c|c|c|c|}
\hline \multirow{2}{*}{$\begin{array}{c}\text { Altura relativa } \\
(\%)\end{array}$} & \multicolumn{3}{|c|}{ Dominantes } & \multicolumn{3}{|c|}{ Codominantes } & \multicolumn{3}{|c|}{ Intermedios } & \multicolumn{3}{|c|}{ Promedio } \\
\hline & Madera & Corteza & Total & Madera & Corteza & Total & Madera & Corteza & Total & Madera & Corteza & Total \\
\hline 10 & 31,7 & 39,5 & 32,2 & 29,2 & 40,3 & 30,3 & 27,6 & 50,6 & 30,0 & 29,5 & 43,5 & 30,8 \\
\hline 20 & 49,3 & 57,8 & 49,9 & 46,7 & 57,4 & 47,8 & 46,3 & 64,0 & 48,2 & 47,4 & 59,7 & 48,6 \\
\hline 25 & 56,4 & 64,3 & 56,9 & 54,1 & 63,9 & 54,9 & 53,8 & 69,1 & 55,4 & 54,8 & 65,8 & 55,7 \\
\hline 30 & 62,7 & 69,6 & 63,1 & 60,9 & 69,9 & 61,8 & 60,7 & 73,7 & 62,1 & 61,4 & 71,0 & 62,3 \\
\hline 33 & 66,9 & 73,3 & 67,4 & 65,1 & 73,1 & 65,7 & 65,0 & 76,5 & 66,2 & 65,7 & 74,3 & 66,4 \\
\hline 40 & 74,2 & 80,0 & 74,6 & 72,7 & 79,4 & 73,4 & 72,9 & 81,6 & 73,8 & 73,3 & 80,4 & 73,9 \\
\hline 50 & 83,2 & 87,2 & 83,4 & 81,3 & 86,6 & 81,6 & 82,3 & 87,5 & 82,8 & 82,2 & 87,1 & 82,6 \\
\hline 60 & 90,0 & 92,4 & 90,2 & 87,6 & 92,0 & 88,1 & 89,4 & 92,3 & 90,2 & 89,0 & 92,2 & 89,3 \\
\hline 67 & 93,6 & 95,0 & 93,6 & 90,2 & 93,5 & 90,3 & 93,1 & 95,1 & 93,3 & 92,3 & 94,5 & 92,4 \\
\hline 70 & 95,0 & 96,0 & 95,1 & 92,2 & 95,8 & 92,5 & 94,5 & 96,1 & 94,7 & 93,9 & 96,0 & 94,1 \\
\hline 75 & 96,8 & 97,3 & 96,8 & 93,5 & 96,9 & 93,6 & 96,6 & 97,7 & 96,7 & 95,6 & 97,3 & 95,7 \\
\hline 80 & 98,0 & 98,4 & 98,0 & 94,5 & 97,9 & 94,9 & 97,8 & 98,4 & 97,9 & 96,8 & 98,2 & 96,9 \\
\hline 90 & 99,5 & 99,6 & 99,3 & 96,1 & 99,4 & 96,5 & 99,4 & 99,6 & 99,5 & 98,3 & 99,5 & 98,4 \\
\hline
\end{tabular}


En todas las clases de copa más del $60 \%$ de la masa seca total de corteza se concentra en la base del árbol (25\% de la altura total) (cuadro 6). Sin embargo, los árboles intermedios poseen un mayor porcentaje de masa seca a una misma altura relativa hasta aproximadamente un tercio de la altura total (figura 2). Desde la mitad del árbol hacia el ápice, aumenta en forma gradual y la curva tiende a estabilizarse desde aproximadamente el $75 \%$ de la altura del árbol. Similar comportamiento presentan el espesor de corteza y la razón de corteza seca; éstos son mayores en las primeras secciones del fuste para luego estabilizarse desde aproximadamente el $75 \%$ de la altura del árbol.

En general, las tres clases de copa concentran más del $50 \%$ de la masa seca total del fuste en el primer cuarto de su altura total, más del $80 \%$ en la primera mitad y más del $90 \%$ en el tercer cuarto (cuadro 6; figura 2).

La masa seca total promedio en las tres clases de copa de madera, corteza y total fuste se concentra principalmente en la primera mitad $(82,2 \%$,
$87,1 \%$ y $82,6 \%$ respectivamente) (cuadro 6; figura 2).

Funciones predictoras de biomasa. Las funciones ensayadas a partir del modelo lineal $Y=b * X$ mostraron mejores valores de $\mathrm{R}^{2}$, EEE y $\mathrm{F}$ que las funciones ensayadas a partir del modelo lineal $Y=a+b * X$, razón por la cual se aplicó el primer modelo a todas las funciones predictoras de biomasa ajustadas.

El dap fue utilizado como variable predictora de masa seca de casi todos los componentes, con excepción de la masa seca de acículas; se utilizó el dap solo $\left(\right.$ como dap $\left.{ }^{2}\right)$ o en combinación con otras variables como altura total (ht) y diámetro en la base de la copa viva (dbc) (cuadro 7).

El dap ${ }^{2}$ mostró una alta correlación con los valores de madera del fuste, total fuste y total árbol, es decir, con los valores del principal componente de biomasa del árbol. En los tres casos, la variable independiente dap ${ }^{2}$ explicó más del 96\% de la variación total de cada componente y mostró altos valores de $\mathrm{F}$ (cuadro 7). Consideran-
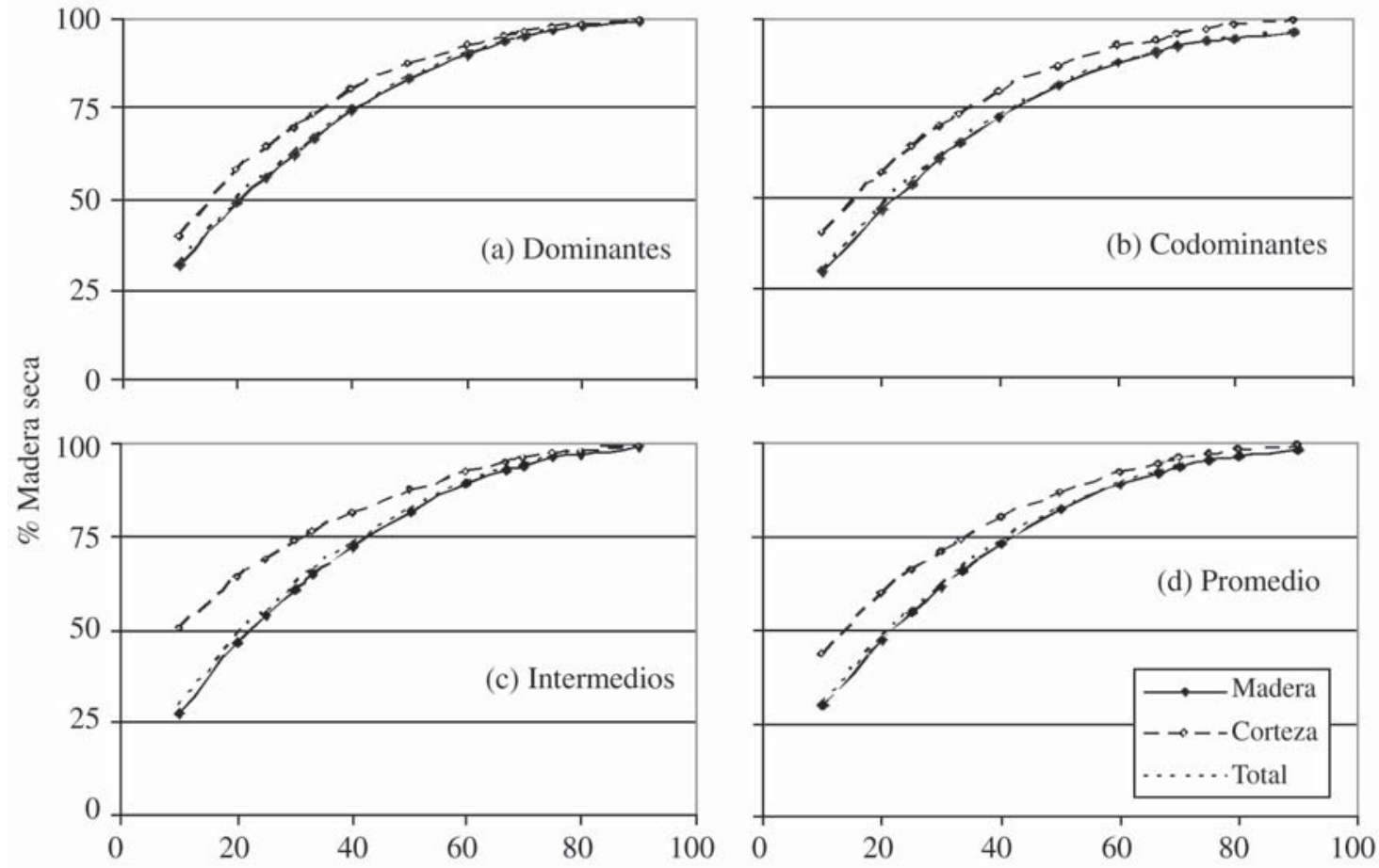

Altura relativa $(\%)$

Figura 2. Distribución de masa seca de madera, corteza y total según altura relativa por clase de copa. Distribution of wooden dry mass, bark and total according to relative height for class of crown. 


\section{CUADRO 7}

Funciones predictoras de biomasa por componente y estadística asociada.

Predictors functions of biomass by component and associate statistic.

\begin{tabular}{|llcrrr|}
\hline Componente $(\mathrm{kg})$ & \multicolumn{1}{c}{ Función } & $\mathrm{R}^{2}$ ajustado $(\%)$ & EEE & $\mathrm{F}$ & $\mathrm{n}$ \\
\hline Madera fuste & $\mathrm{M}=0,486798568 *$ dap $^{2}$ & 96,8 & 112,4 & 1593,2 & 11 \\
Corteza fuste & $\mathrm{M}=0,047428414 *$ dap $*$ ht & 58,7 & 28,9 & 162,6 & 11 \\
Total fuste & $\mathrm{M}=0,526000030 *$ dap $^{2}$ & 96,6 & 122,3 & 1570,7 & 11 \\
Acículas & $\mathrm{M}=0,011092764 *$ dat $* \mathrm{c}$ & 88,8 & 2,2 & 380,6 & 12 \\
Ramillas & $\mathrm{M}=0,000113863 *$ dap $^{2} * \mathrm{dbc}$ & 85,4 & 2,4 & 227,9 & 12 \\
Ramas & $\mathrm{M}=0,000303168 *$ dap $^{2} * \mathrm{dbc}$ & 92,1 & 5,1 & 346,0 & 12 \\
Total copa * & $\mathrm{M}=0,002308930 *$ dap $^{2} * \mathrm{dbc}$ & 85,3 & 66,1 & 119,5 & 11 \\
Total árbol $* *$ & $\mathrm{M}=0,544853393 *$ dap $^{2}$ & 96,7 & 128,3 & 1532,1 & 11 \\
\hline
\end{tabular}

\footnotetext{
*: Biomasa total de la copa incluye la sección del fuste de la copa.

**: Biomasa total del árbol sobre el suelo.

M: Masa seca $(\mathrm{kg})$ del componente.

dap: Diámetro a la altura del pecho $(\mathrm{cm})$.

dat: Diámetro a la altura del tocón $(\mathrm{cm})$.

dbc: Diámetro en la base de la copa viva $(\mathrm{cm})$.

ht: Altura total (m).

lc: Longitud de copa viva (m).

$\mathrm{R}^{2}$ : Coeficiente de determinación ajustado.

EEE: Error estándar de estimación.

F: Valor prueba F.

n: Número de unidades muestrales.
}

do que el dap es una variable de fácil medición en terreno y pensando en una posible aplicación de estas funciones a una cuantificación de biomasa en una gran extensión de plantaciones, se puede afirmar que es positivo que el dap sea la única variable a utilizar para la estimación de los valores de biomasa fustal.

En el ajuste de funciones de componentes de la copa, el diámetro en la base de la copa viva (junto con el dap) fue utilizado como variable predictora en casi todas las funciones, con excepción de la función de masa seca de acículas. Específicamente, las funciones ajustadas con la variable dap ${ }^{2 *} \mathrm{dbc}$ mostraron valores de $\mathrm{R}^{2}$ sobre $85 \%$ (92\% en el caso de la función de masa seca de ramas) y valores muy bajos de EEE en comparación a los valores obtenidos en las funciones de componentes fustales, lo que indica una baja dispersión respecto de la recta de regresión de $Y$ sobre $X$ (cuadro 7 ).

En la función de masa seca de acículas, la variable predictora equivalente al producto del diámetro a la altura del tocón (dat) por la longitud de la copa viva (lc) fue la que explicó en mayor porcentaje la variación de este componente de la copa y mostró además el menor valor de EEE de todas las funciones ajustadas (cuadro 7).

\section{CONCLUSIONES}

La madera es el componente que más aporta a la biomasa total del árbol $(88,9 \%)$, seguido de la corteza $(8,2 \%)$, ramas $(1,5 \%)$, acículas $(0,8 \%)$ y ramillas $(0,7 \%)$. Existen diferencias significativas entre las tres clases de copa en la masa seca de madera, ramas y total del árbol.

La copa presenta una distribución irregular de la masa seca a lo largo de su extensión; los árboles dominantes poseen copas de mayor longitud, con ramas más gruesas y de mayor longitud, lo que está directamente relacionado con los valores de biomasa de cada componente de la copa y del total de la copa.

El mayor porcentaje de masa seca del fuste se concentra en las secciones basales del árbol: el $55,7 \%$ de la masa seca total en el primer cuarto, el $82,6 \%$ en la mitad y el $95,7 \%$ en el tercer cuar- 
to, tras lo cual el aumento de masa seca disminuye gradualmente.

El dap fue utilizado como variable predictora de masa seca de casi todos los componentes, con excepción de la masa seca de acículas; se utilizó el dap solo (como dap ${ }^{2}$ ) o en combinación con otras variables como altura total y diámetro en la base de la copa viva.

El diámetro en la base de la copa viva y la longitud de copa viva fueron las variables que en mayor porcentaje explicaron la variación de los valores de los componentes de la copa. Al ser ambas características de la copa, están directamente relacionadas con la biomasa de sus componentes; sin embargo, son variables de difícil medición en árboles en pie, por lo que podríamos pensar en una estimación del dbc a partir del dap (considerando que ambas son variables del fuste), para disponer de los valores que necesitamos para la estimación de los componentes del árbol usando las funciones ajustadas en este estudio.

Estas funciones podrían ser utilizadas en rodales de la misma especie localizados en otros sitios o de diferentes edades a las del rodal en estudio, sin embargo, se requiere comprobar si las funciones son aplicables a otra zona. Se debe hacer un muestreo de comprobación (también conocido como "validación"), en donde se deben utilizar datos independientes del modelo para ajustarlo o comprobar si sirve en condiciones distintas a las originales.

\section{BIBLIOGRAFIA}

(1) DE LA MAZA, F. Variación de biomasa en ensayos de clareos de Eucalyptus globulus Labill. VIII Región. $1^{\mathrm{a}}$ ed., Santiago: Universidad de Chile, 1991, 95 p.

(2) SÁEZ, M. Biomasa y contenido de nutrientes de renovales no intervenidos de roble-raulí. $1^{\text {a }}$ ed., Santiago: Universidad de Chile, 1991, 103 p.

(3) BAQUERO, J., M.J. MORENO. La biomasa como fuente de energía renovable. En: Sitio web de energía. 15 marzo 2000. citado 24 febrero 2004]. Disponible en: <http:/ /www.energuia.com/es/bibliografia3.aspx?ID=755>.

(4) OGIER, J., D. BALLERINI, J. LEYGUE, L. RIGAL, J. POURQUIE. Ethanol production from lignocellulosic biomass. Oil \& Gas Science and Technology-Revue De L 'Institut Français du Petrole (France), 1999, vol. 54, $\mathrm{N}^{\circ} 1$, p. 67-94.

(5) RITSON, P., S. SOCHACKI. Measurement and prediction of biomass and carbon content of Pinus pinaster trees in farm forestry plantations, south western
Australia. Forest Ecology and Management, 2003, vol. 175, p. 103-117.

(6) LACLAU, P. Biomass and carbon sequestration of ponderosa pine plantations and native cypress forests in northwest Patagonia. Forest Ecology and Management, 2003, vol. 180 , p. 317-333.

(7) FLORES, F.J., H.L. ALLEN. Efectos del clima y capacidad de almacenamiento de agua del suelo en la productividad de rodales de pino radiata en Chile: un análisis utilizando el modelo 3-PG. Bosque (Chile), 2004, vol. $25, \mathrm{~N}^{\mathrm{o}} 3$, p. 11-24.

(8) RICHARDSON, B., M. SKINNER, G. WEST. The role of forest productivity in defining the sustainability of plantation forests in New Zealand. Forest Ecology and Management, 1999, vol. 122, p. 125-137.

(9) LINEROS, M. Funciones locales de biomasa (madera y corteza) para Pinus radiata D. Don. VIII Región. $1^{a}$ ed., Chillán: Universidad de Concepción, 1986, 67 p.

(10) BLAZIER, M., T. HENNESSEY, T. LYNCH, R. WITTWER. Comparison of branch biomass relationships for North Carolina and Oklahoma/Arkansas loblolly pine seed sources growing in southeastern Oklahoma. Forest Ecology and Management, 2002, vol. 159, p. 241-248.

(11) WATT, M., D. WHITEHEAD, E. MASON, B. RICHARDSON, M. KIMBERLEY. The influence of weed competition for light and water on growth and dry matter partitioning of young Pinus radiata, at a dryland site. Forest Ecology and Management, 2003, vol. 183, p. 363-376.

(12) ZHANG, Y. A physiologically based simulation model for loblolly pine. $1^{\text {a }}$ ed, Athens, GA: University of Georgia, 1997, 127 p.

(13) BANDARA, G., D. WHITEHEAD, D. MEAD, D. MOOT. Effects of pruning and understorey vegetation on crown development, biomass increment and aboveground carbon partitioning in Pinus radiata D. Don trees growing at a dryland agroforestry site. Forest Ecology and Management, 1999, vol. 124, p. 241-254.

(14) SANTIBÁÑEZ, F., J. URIBE. Atlas agroclimático de Chile, regiones sexta, séptima, octava y novena. $5^{\mathrm{a}}$ ed., Santiago: Corfo, Ministerio de Agricultura, 1993, 73 p.

(15) SMITH, D.M. The practice of silviculture. $3^{\text {rd }}$ ed., New York: Wiley, 1986, 242 p.

(16) RODRIGUEZ, R., M. ESPINOSA, G. HOFMANN, M. MARCHANT. Needle mass, fine root and stem wood production in response to silvicultural treatment, tree size and competitive status in radiata pine stands. Forest Ecology and Management, 2003, vol. 186, p. 287-296.

(17) RODRIGUEZ, R., G. HOFMANN, M. ESPINOSA, D. RIOS. Biomass partitioning and leaf area of Pinus radiata trees subjected to silvopastoral and conventional forestry in the VI region, Chile. Revista Chilena de Historia Natural (Chile), 2003, vol. 76, No 3, p. 437-449.

(18) HELMISAARI, H., K. MAKKONEN, S. KELLOMÄKI, E. VALTONENC, E. MÄLKÖNEN. Below- and aboveground biomass, production and nitrogen use in Scots pine stands in eastern Finland. Forest Ecology and Management, 2002, vol. 165, p. 317-326.

(19) HUSCH, B., C. MILlER, T. BEERS. Forest Mensuration. $2^{\text {nd }}$ ed., New York: Wiley. 1972. 667 p.

(20) CANOVOS, G. Probabilidad y estadística. Aplicaciones y métodos. $2^{\mathrm{a}}$ ed., México D.F.: Mc Graw-Hill, 1988. 554 p.

(21) GERDING, V., J.E. SCHLATTER. Variables y factores del sitio de importancia para la productividad de Pinus radiata D. Don en Chile. Bosque (Chile), 1995, vol. 16, $\mathrm{N}^{\mathrm{o}}$ 2, p. 39-56. 